$10-1-2020$

\title{
Caseous mitral valve calcification and concurrent hypertrophic obstructive cardiomyopathy: A rare cause of stroke
}

\author{
Waqas Ullah \\ Thomas Jefferson University \\ Donald C. Haas \\ Thomas Jefferson University \\ Talal Almas \\ Royal College of Surgeons in Ireland
}

Aws- Al-Mukhtar

Imperial College London

Asad Inayat

Khyber Teaching Hospital

Follow this and additional works at: https://jdc.jefferson.edu/cardiologyfp

Part of the Cardiology Commons

Let us know how access to this document benefits you

\section{Recommended Citation}

Ullah, Waqas; Haas, Donald C.; Almas, Talal; Al-Mukhtar, Aws-; and Inayat, Asad, "Caseous mitral valve calcification and concurrent hypertrophic obstructive cardiomyopathy: $A$ rare cause of stroke" (2020). Division of Cardiology Faculty Papers. Paper 68.

https://jdc.jefferson.edu/cardiologyfp/68

This Article is brought to you for free and open access by the Jefferson Digital Commons. The Jefferson Digital Commons is a service of Thomas Jefferson University's Center for Teaching and Learning (CTL). The Commons is a showcase for Jefferson books and journals, peer-reviewed scholarly publications, unique historical collections from the University archives, and teaching tools. The Jefferson Digital Commons allows researchers and interested readers anywhere in the world to learn about and keep up to date with Jefferson scholarship. This article has been accepted for inclusion in Division of Cardiology Faculty Papers by an authorized administrator of the Jefferson Digital Commons. For more information, please contact: JeffersonDigitalCommons@jefferson.edu. 


\section{Caseous mitral valve calcification and concur- rent hypertrophic obstructive cardiomyopathy: A rare cause of stroke}

Mitral annulus calcification (MAC) is a relatively common condition with a prevalence of about $14 \%$, often seen at old age and results from degenerative changes in the mitral valve fibrous skeleton [1]. An exceedingly rare and adverse variant of MAC is the caseous calcification of the mitral annulus (CCMA), which accounts for only $0.6 \%$ of all MAC cases [2]. It can be easily confused with tumors, vegetations, or cysts; thus, often underdiagnosed and mistreated. CCMA in association with other comorbidities can potentially increase the risk of cerebrovascular accidents (CVA). Here, we present the first case of CCMA with concomitant hypertrophic obstructive cardiomyopathy (HOCM) presenting with cardioembolic stroke complicated by hemorrhagic conversion.

\section{Case presentation}

A 68-year-old woman with a past medical history of hypertension and HOCM presented to the emergency department (ED) with sudden left-sided facial weakness and slurred speech for the last $2 \mathrm{~h}$. She also complained of sudden onset of dizziness and difficulty concentrating, while she was working from home, attempting to sign into a zoom meeting. She noted that she was not able to sign in, and was having trouble with her coordination and typing on her computer.

In the $\mathrm{ED}$, she was afebrile with a temperature of $98 \mathrm{~F}$, tachycardiac (heart rate 120 ) and hypotensive (90/60 $\mathrm{mmHg}$ ). Her oxygen saturation ( $\mathrm{SaO} 2)$ was $85 \%$ on room air. Her physical examination was significant for right middle lung rhonchi, facial droop and garbled speech. Her National Institutes of Health Stroke Scale (NIHSS) was 4 . Her cardiovascular examination revealed a pansystolic murmur at the mitral area.

Laboratory investigation was significant for leukocytosis (white blood cells count of $17.4 \times 109 / \mathrm{L}$ ). Rest of the blood work was unremarkable. Her nasal swab real-time fluorescence polymerase chain reaction (RT-PCR) for severe acute respiratory syndrome coronavirus-2 (SARS-CoV-2) returned negative. The computed tomography (CT) head ruled out hemorrhagic stroke. Her brain magnetic resonance imaging (MRI) was consistent with bihemispheric cortical and subcortical embolic strokes. Electrocardiogram (EKG) showed normal sinus rhythm. Transthoracic echocardiogram (TTE) revealed a hyperdynamic anterior ventricle, systolic anterior motion of the mitral valve, peak resting left ventricular outflow tract (LVOT) gradient of $84 \mathrm{mmHg}$ and posterior mitral leaflet mass suggestive of vegetation complicated by moderateto-severe mitral regurgitation. Her cardiac CT showed posterior MV leaflet calcification (Fig. 1). Cardiac MRI was consistent with
LVEF of $82 \%$; focal HOCM with LVOT obstruction and systolic anterior motion of the mitral valve leaflet and moderate mitral valve regurgitation (Figs. 2, 3) There was no delayed myocardial enhancement to suggest scar, infarct, or infiltrative disorder.

She was placed on supplemental oxygen at $2 \mathrm{~L} / \mathrm{min}$ via nasal cannula and started on antibiotics for aspiration pneumonia. She was emergently treated with tissue plasminogen activator (tPA), suspecting cerebrovascular accident (CVA) in the right middle cerebral artery (MCA) territory. She was admitted to the neurocritical unit for close neurological monitoring, stroke workup, and further medical management.

Patient developed a sudden onset of headaches 6 h later. Her neurological exam remained unchanged. A repeat $\mathrm{CT}$ head revealed a large right frontal and temporal intracerebral hemorrhage associated with right-sided subarachnoid hemorrhage (SAH) and right to left midline shift. She was managed with supportive care and started on a nicardipine drip. She also received hypertonic saline for osmotic diuresis for goal sodium level of $155-160 \mathrm{mEq} / \mathrm{L}$ for cerebral edema. She received a loop recorder and was ultimately discharged to a rehabilitation center with residual weakness. On follow-up, the biotel cardionet monitor recorded no episodes of $\mathrm{AF}$, heart block, pauses, or other arrhythmias.

\section{Discussion}

CCMA is a distinctive and special variant of MAC that results from liquefactive necrosis of the posterior annulus of the mitral valve and is usually considered a benign condition [1,2]. In rare cases, however, it may compromise the normal function of the mitral valve, subsequently causing regurgitation or mitral stenosis [3]. The later can cause significant left ventricular inflow tract obstruction. Other rare valvular complications of CCMA may also predispose patients to left ventricular outflow obstruction (LVOT), atrial fibrillation (AF), and aortic valve abnormalities [4]. It can also lead to the same hemodynamic consequences of constrictive pericarditis. While all these complications significantly increase the risk of CVA, we believe that CCMA independently carries a higher risk of cerebrovascular complications especially in presence of HOCM $[1,3,5,6]$.

A review of 130 patients with CCMA reported a $19.2 \%$ prevalence of CVAs, significantly higher than seen with MAC alone (11.8\%) [7]. This highlights the importance of differentiating CCMA from MAC to prevent dire consequences. The suggestive echocardiographic findings of CCMA include areas of decreased echogenicity, a central echolucent zone, and the absence of acoustic shadowing. Microscopically, CCMA is characterized by liquefaction necrosis with amorphous eosinophilic acellular material surrounded by lymphocytes and macrophages amid multiple small calcifications [1]. Intriguingly, only four of the reported 25 (16.0\%) patients with CCMA who suffered a CVA had a history of 


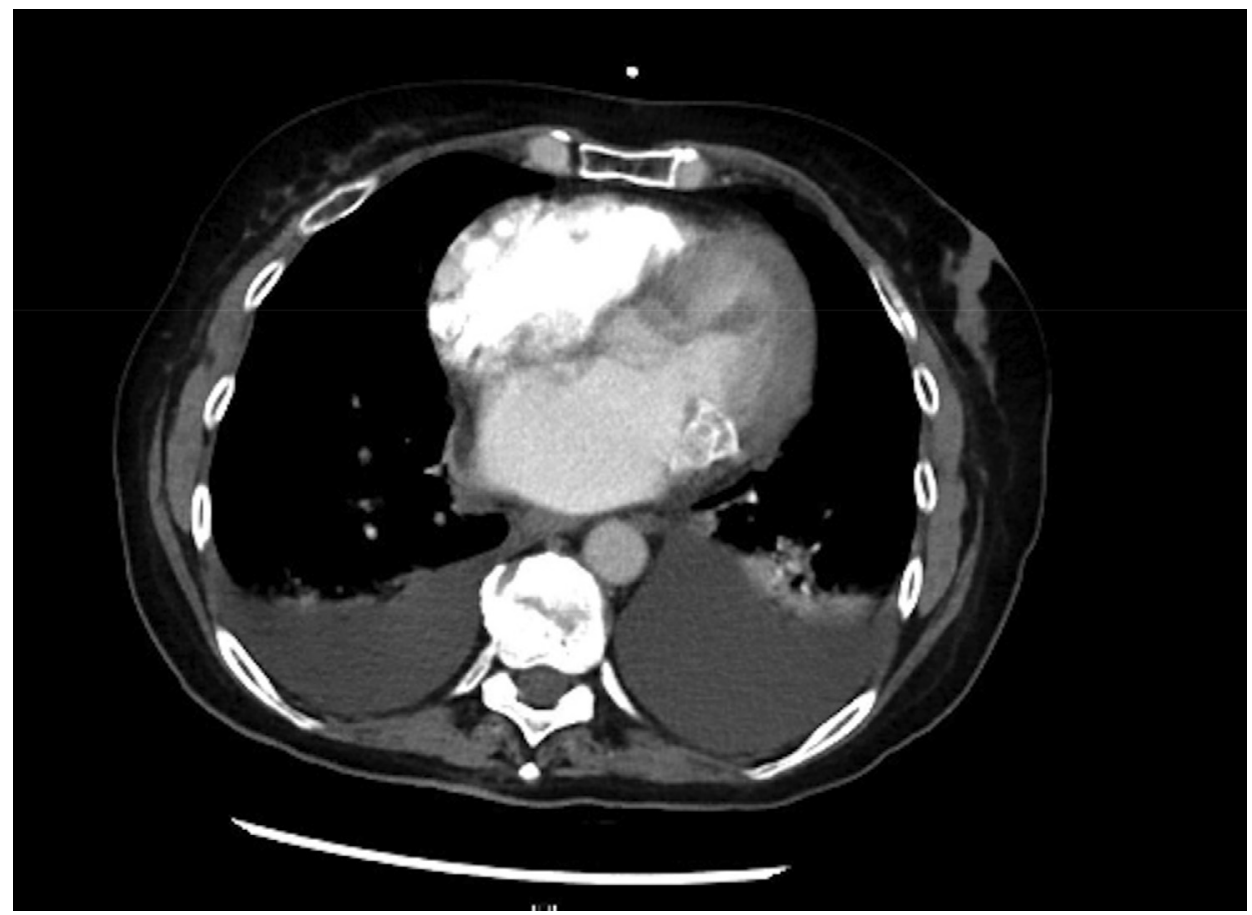

Fig. 1. CT scan showing pleural effusion and mitral annular calcification.

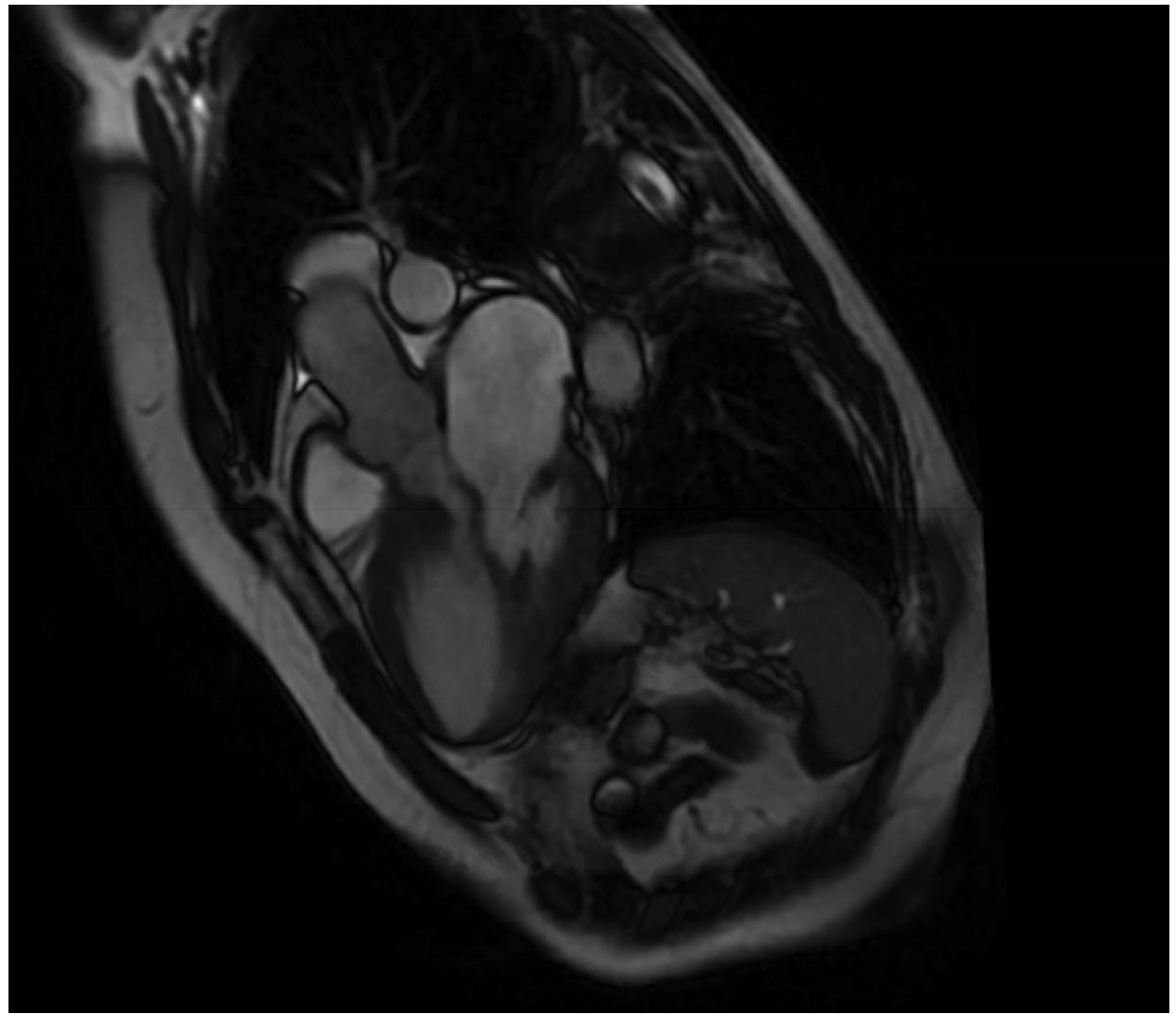

Fig. 2. Coronal section of cardiac MRI showing LVOT obstruction and mitral annular calcification.

AF. This further indicates that CCMA might serve as an independent risk factor for CVA.

The risk of stroke in patients with HOCM alone is merely $1.0 \%$, which is largely attributed to the high rate of AF in these patients
[8]. However, in our case, a biotel cardionet monitor recorded no episodes of AF indicating that the hemodynamic instability coupled with cardiac embolization was the repercussions elicited by CCMA. We believe that embolization of the caseous necrotic debris 


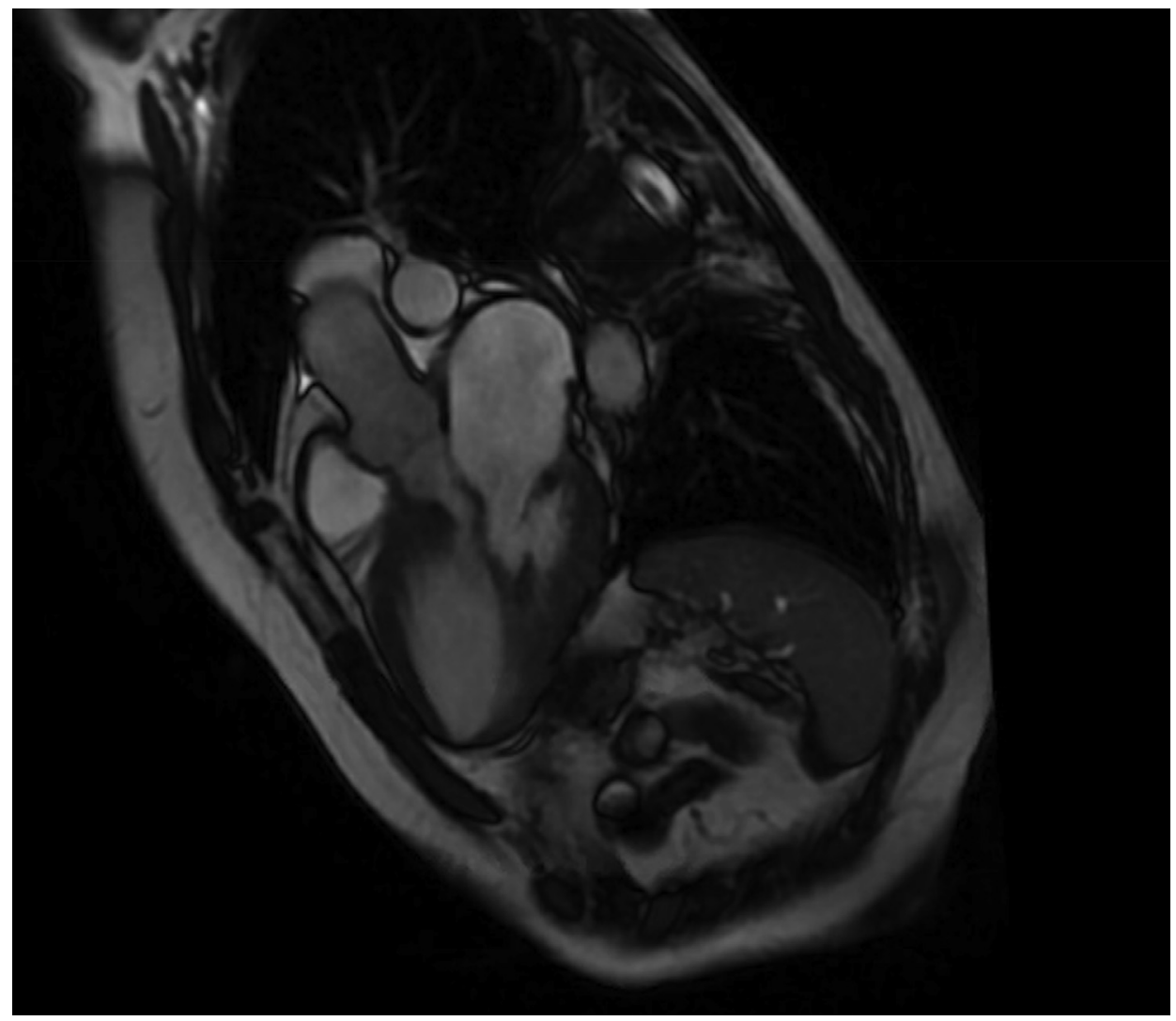

Fig. 3. Sagittal section of cardiac MRI showing LVOT obstruction and mitral annular calcification.

of the mitral annulus was the major cause of multifocal stroke in our case. The stroke risk in our case was further precipitated by the LVOT obstruction induced hemodynamic instability in presence of HOCM.

Our case also highlights the importance of early recognition of this potentially fatal condition. Diagnosing CCMA is itself an onerous diagnostic challenge due to its rarity and perplexing clinical manifestations. Prompt detection and management of CCMA and HOCM may aid in foiling adverse outcomes, such as CVA. Lesions afflicting the posterior mitral annulus on echocardiography should be evaluated through further imaging to rule out CCMA so that the grave ramifications elicited by it can be prevented. In our case, a definitive diagnosis was made with MRI and CT scan which ruled out other causes and confirmed the presence of CCMA. This necessitates the need for multimodality imaging to yield a definitive diagnosis of CCMA. The patient also developed moderate to severe mitral regurgitation due to prolonged CCMA. Previous studies have shown that mitral regurgitation develops secondary to longstanding MAC [9]. This grave valvular abnormality, along with the patient's history of HOCM and LVOT culminated in hemodynamic instability and CVA.

It is unclear whether treating patients with CCMA prophylactically with valve replacement can reduce the risk of stroke. Also, the risk of tPA associated hemorrhagic conversion of embolic stroke in these patients warrants more studies on a larger scale. Current studies focused on the imperativeness of conservative management in treating early CCMA by controlling other cardiovascular comorbidities (hypertension, dyslipidemia) and prevention of complications such as arrhythmias $[10,11]$. Antithrombotic therapy is reserved for patients with CVA and cardioembolic complications. Patients with symptomatic severe mitral regurgitation and mitral stenosis are treated with surgical mitral valve replacement as percutaneous mitral balloon valvuloplasty is generally not effective. Due to a high risk of operative mortality a careful riskbenefit evaluation is recommended.

\section{Conclusion}

In our case, CCMA along with concurrent HOCM and LVOT obstruction resulted in hemodynamic instability leading to cardioembolic stroke and subsequent hemorrhagic conversion with tPA. We, therefore, advocate for early recognition and consideration of elective surgical resection of CCMA in patients with HOCM, even in asymptomatic patients who are good surgical candidates. Nevertheless, more studies are required to understand the pathogenesis and the precise clinical course of CCMA in patients with HOCM.

\section{Learning objectives}

CCMA along with concurrent HOCM and LVOT obstruction can result in hemodynamic instability, leading to cardioembolic stroke and subsequent hemorrhagic conversion with tPA. Early recognition and consideration of elective surgical resection of CCMA in patients with HOCM, even in asymptomatic patients who are good surgical candidates, therefore remains pivotal in avoiding potential complications

\section{Funding}

None.

\section{Declaration of Competing Interest}

The authors report no relationships that could be construed as a conflict of interest. 


\section{Acknowledgements}

None.

\section{References}

[1] A. Goldberg, G. Singh, S. Boateng, M. Tracy, Caseous calcification of the mitral annulus causing multiple cardioembolic strokes, J. Am. Coll. Cardiol. 67 (13 Supplement) (2016 Apr 5) 1184.

[2] H. Arora, P. Madan, L. Simpson, R.F. Stainback, Caseous Calcification Of The Mitral Annulus, Tex Hear Inst. J. 35 (2) (2008) 211-213.

[3] E. Curl, E. Riemer, Caseous calcification of the mitral annulus : case report and brief review, Eur Heart J. 2 (2018) 1-5.

[4] C. Report, K. Liu, J. Hollman, N. Deumite, Caseous mitral annular calcification presenting as a stroke: a cracking of the shell, Ann. Atheroscler. Res. [Internet] 1 (2) (2018) $1-4$

[5] G. Minardi, C. Manzara, G. Pulignano, P.G. Pino, H. Pavaci, M. Sordi, et al, Caseous calcification of the mitral annulus with mitral regurgitation and impairment of functional capacity: A case report, J. Med. Case Rep. 2 (2008) 47.

[6] D. Kim, C. Shim, G.R. Hong. H. Jeong JW. Ha, Morphological and functional characteristics of mitral annular calcification and their relationship to stroke, PLOS ONE 15 (1) (2020) 1-11.

[7] C.A. Dietl, C.M. Hawthorn, V. Raizada, Risk of cerebral embolization with caseous calcification of the mitral annulus, Open Cardiovascular Med. J. 10 (2016) 221

[8] S. Haruki, Y. Minami, N. Hagiwara, Stroke and embolic events in hypertrophic cardiomyopathy, Stroke 47 (2016) 936-942.

[9] N. Mallat, M. Limeme, H. Zaghouani, F. Mootemri, S. Majdoub, H. Amara, et al, Caseous calcification of the mitral annulus on MDCT: a rare intracardiac mass, Acta Radiol. Short Reports 2 (7) (2013), 204798161350217.
[10] Y. Okada, Surgical management of mitral annular calcification, Gen. Thorac. Cardiovasc. Surg. 61 (11) (2013 Nov) 619-625.

[11] L.S. Fong, A.J. McLaughlin, N.L. Okiwelu, I.A.J. Nordstrand, M. Newman, J. Passage, et al, Surgical management of caseous calcification of the mitral annulus, Ann. Thorac. Surg. 104 (3) (2017 Sep) e291-e293.

Waqas Ullah *

Donald Haas

Abington Hospital - Jefferson Health, Abington, PA, United States * Corresponding author. E-mail address: waqasullah.dr@gmail.com (W. Ullah)

Talal Almas Royal College of Surgeons in Ireland, Dublin, Ireland

Aws- Al-Mukhtar Imperial College London, London, United Kingdom

Asad Inayat

Khyber Teaching Hospital, Peshawar, Pakistan

Received 5 September 2020

Received in revised form 12 September 2020 Accepted 16 September 2020

Available online 29 September 2020 